\title{
PREFACE
}

\section{Speech Pathologists in Long-Term Care}

This issue brings together articles that discuss the knowledge base and skill set needed to work in long term care. To be a successful clinician in this setting, one must possess more than an understanding of communication and swallowing disorders. It is essential that speechlanguage pathologists work effectively with a variety of other health care professions, can counsel families, are informed about all state and federal regulations, consistently and accurately document their services, are informed about the effects of medications, feel at ease with older adults, and most importantly, collaborate with the older adult to help them live as they choose. It's an exciting time to be working in long term care as approaches to the delivery of care are changing dramatically. Smaller care communities are being created that allow elders to live with a small number of other residents in an environment that resembles a house more than a hospital. Residents have been empowered to choose their dining and sleep schedules, have pets and children on the premises, and take on responsibilities and chores such as gardening and housekeeping. All around the country the focus is shifting from the provision of good healthcare to an approach that attends to the psychosocial, emotional, and spiritual well-being of each individual. Everything from staff training, to organizational structure, to the physical design of the setting is being examined and reorganized. Speech-language pathologists have an important role to play in this person-centered care movement that advocates for a physical and social environment and care delivery system focused on the values of choice, dignity, respect, self-determination and purposeful living.
We should begin by providing sufficient education to our graduate and undergraduate students. Mahendra, Freemont, and Dionne share their innovative approach for providing speech language pathology students with exposure to long-term care and clinical training in service delivery for persons with dementia. Their program leads students through distinct stages of creating awareness and a foundation of knowledge, teaching clinical skills, hands on practice, and ongoing self-reflection. Through this exposure, students' attitudes about with people with dementia improved. This is significant as people with dementia are a fast growing clinical population. In the next article, Johnson reminds us about how detrimental medication side effects can be to our clients' cognitive status. He cautions that many older adults take multiple medications that may have a direct effect on the success of rehabilitation efforts. Collaboration with pharmacists and physicians will lead to better awareness of medication reactions and improved outcomes for communication and swallowing interventions.

In the article by Casper, she provides us with a look into the day of a speech-language pathologist in the long term care setting. It quickly becomes clear to the reader that an effective clinician must be willing to wear many hats!

More than half of the speech-language pathologist case load in long term care is clients who require dysphagia services. Mealtime is more than an occasion to consume nutrients; it's an important opportunity for socialization, celebration, choice, and comfort. These aspects of mealtime are often more important to our
${ }^{1}$ I.D.E.A.S., Inc., Kirtland, Ohio.

Address for correspondence: Jennifer A. Brush, M.A., CCC-SLP, I.D.E.A.S., Inc., 8055 Chardon Rd., Kirtland, OH 44094 (e-mail: jbrush@IDEASConsultingInc.com).

Speech Pathologists in Long-Term Care; Guest Editor, Jennifer A. Brush, M.A., CCC-SLP
Semin Speech Lang 2013;34:3-4. Copyright (C) 2013 by Thieme Medical Publishers, Inc., 333 Seventh Avenue, New York, NY 10001, USA. Tel: +1(212) 584-4662. DOI: http://dx.doi.org/10.1055/s-0033-1341350. ISSN 0734-0478. 
clients than if they are able to swallow correctly. Bowman discusses the adaptation and implementation of the New Dining Practice Standards published by the Pioneer Network and Food and Dining Clinical Standards Task Force in 2011. These standards support individualized care and self-directed living versus traditional diagnosis-focused treatment for people living in long term care settings. Speech-language pathologists have a unique and critical role in advocating for resident rights and quality of life from a wellness perspective in addition to a medical perspective.

Finally Bruce, Brush, Sanford, and Calkins look at the physical environment as a therapeutic tool. Research recognizes that dementia-related communication impairment can be exacerbated by environmental barriers such as inadequate lighting, noisy conditions, poor or absent environmental cues, and visual clutter. The authors stress that speech-language pathologists should address these environmental barriers as part of a comprehensive treatment plan for clients with dementia.

It is hoped that this review of topics pertinent to the long term care setting with encourage the reader to expand their current practices to include new care delivery methods that lead to better outcomes for elders.

Jennifer A. Brush, M.A., CCC-SLP ${ }^{1}$
Guest Editor 